disorders: National Institute of Mental Health report. Nicotine Tob Res 2008; 10: 1691-715.

9 Munafò MR, Hitsman B, Rende R, Metcalfe C, Niaura R. Effects of progression to cigarette smoking on depressed mood in adolescents: evidence from the National Longitudinal Study of Adolescent Health. Addiction 2008; 103 162-71.

10 Munafò MR, Araya R. Cigarette smoking and depression: a question of causation. Br J Psychiatry 2010; 196: 425-6.

Vanessa Argondizo dos Santos, School of Medicine, Pontificia Universidade Católica Rio Grande do Sul; Ana Maria Migott, School of Medicine, Universidade de Passo Fundo; Claiton Henrique Dotto Bau, Department of Genetics, Instituto de Biociências, Universidade Federal do Rio Grande do Sul; José Miguel Chatkin, Av. Ipiranga 6690, room 315, 3rd floor, School of Medicine Graduation Office, Pontificia Universidade Católica Rio Grande do Sul, Porto Alegre 90610-000, Brazil. Email: jmchatkin@pucrs.br

doi: 10.1192/bjp.197.5.413

\section{Does medication benefit the long-term psychiatric outcomes of children with ADHD?}

Langley and colleagues ${ }^{1}$ reported 5-year follow-up outcomes of young children with attention-deficit hyperactivity disorder (ADHD) and the maternal and social factors related to the prognosis. The findings provide evidence of high comorbidity of antisocial behaviours associated with ADHD, drawing attention to the long-term outcomes of the disorder. Yet, in my opinion, additional information needs to be clarified regarding the findings.

The authors showed that medication use was not significantly associated with conduct disorder diagnosis or other antisocial behaviours. However, this interesting result was not discussed in detail in the article. What I am interested in is whether medication could reduce the risk of developing psychiatric diseases. Recently, studies have shown that treatment with stimulant drugs for ADHD could reduce the risk for some psychiatric disorders. In a systematic review, Wilens et $a l^{2}$ reported that medication in childhood was associated with a reduction in the risk for subsequent substance misuse. Biederman et $a l^{3}$ showed that stimulant treatment of youths with ADHD decreased the risk for depressive and anxiety disorders and disruptive behaviour later in life. Both studies indicate that medication can benefit psychiatric outcomes. In Langley et al's study, ${ }^{1}$ most of the participants $(63 \%)$ received prescribed stimulant drugs, but the psychological outcomes were not optimistic regarding the prognosis of conduct disorder. Does this result suggest that medication is not beneficial for children with ADHD in the long term? What can account for it? In addition, why did children who were prescribed medication have more ADHD symptoms than those no longer using medication? ${ }^{1}$

1 Langley K, Fowler T, Ford T, Thapar AK, van den Bree M, Harold G, et al Adolescent clinical outcomes for young people with attention-deficit hyperactivity disorder. Br J Psychiatry 2010; 196: 235-40.

2 Wilens TE, Faraone SV, Biederman J, Gunawardene S. Does stimulant therapy of attention-deficit/hyperactivity disorder beget later substance abuse? A meta-analytic review of the literature. Pediatrics 2003; 111: 179-85.

3 Biederman J, Monuteaux MC, Spencer T, Wilens TE, Faraone SV. Do stimulants protect against psychiatric disorders in youth with ADHD? A 10-year follow-up study. Pediatrics 2009; 124: 71-8.

Rongwang Yang, MD, Department of Child Psychology, The Children's Hospital, Zhejiang University School of Medicine, China. Email: colortea@zuaa.zju.edu.cn

doi: 10.1192/bjp.197.5.414

Authors' reply: We agree that the influence of prescribed medication on the long-term psychological outcomes associated with ADHD is an interesting and important area of research. However, we regret that our study is not best placed to address these issues.

Our study utilised a naturalistic design, identifying children recently diagnosed with ADHD through child and adolescent mental health services and paediatric clinics in the UK. As such, no restrictions or controls were placed on the prescription or continuation of stimulant medication in this group. To adequately test the questions posed by Dr Yang, specifically designed trials are required - well beyond the scope of our article.

Our findings indicated that prescription of medication at follow-up was associated with higher rates of ADHD symptoms, but not with the other psychological outcomes we assessed (including conduct disorder and substance use). Because our study does not provide sufficient data on stimulant use over time and because the majority $(90 \%)$ were prescribed stimulant medication at some point, we did not expand further on the reasons for these findings, nor can we speculate on why those prescribed medication at follow-up had more ADHD symptoms.

We are therefore grateful to Dr Yang for highlighting this important area for research, but regret that we cannot address these queries using our data.

\footnotetext{
Kate Langley, Department of Psychological Medicine and Neurology, School of Medicine, Cardiff University, Heath Park, Cardiff, CF14 4XN, UK. Email: langleyk@cf.ac.uk; Tom Fowler, Department of Psychological Medicine and Neurology, School of Medicine, Cardiff University; Tamsin Ford, Child Health Group, Peninsula Medical School, University of Exeter; Ajay K. Thapar, Michael J. Owen, Michael C. O'Donovan, Anita Thapar, Department of Psychological Medicine and Neurology, School of Medicine, Cardiff University, UK

doi: 10.1192/bjp.197.5.414a
}

\section{Erasing trauma memories}

Recent elegant research has raised the salient issue of altering traumatic memories and its treatment implications. Kindt et al suggest that if emotional memory could be weakened or even erased, then we might be able to eliminate the root of many psychiatric disorders, such as post-traumatic stress disorder' In a similar vein, Schiller et $a l^{2}$ reported that 'fearful memories can be wiped out for at least a year using a drug-free technique'. The prospect of erasing distressing memories is indeed compelling and has led to widespread media coverage.

However, this issue elicits important ethical and clinical considerations: first, would we want to erase trauma memories, and second, is it clinically helpful to erase such memories?

Loss of knowledge about the past or oneself may be ethically problematic, although reducing suffering clearly may take precedence. ${ }^{3}$ Our sense of self is constructed from autobiographical memories, and the authenticity of how they link and our trust in this narrative is important for well-being. Furthermore, losing memory can compromise a victim's ability to provide legal evidence: autonomy and beneficence may trump justice, but it would be better if the evidence could be used and the victim did not suffer.

Paradoxically, erasing memories of trauma may not in itself reduce suffering and could even lead to the reverse. In clinical cases where explicit memory of an event has been lost, for example owing to a severe head injury or drug rape (e.g. via flunitrazepam), extreme distress can ensue. The clinical literature suggests that avoidance of trauma memories is associated with worse rather than improved outcome.

We note that the data in the above papers do not in fact indicate memory 'erasure'. Rather, both studies found that fear 